\title{
Prognostic serum biomarkers for colorectal cancer - Review
}

Yovcho Yovchev ${ }^{1}$, Emil Enchev ${ }^{1, *}$, Evgeni Dimitrov ${ }^{1}$, Georgi Minkov ${ }^{1}$ and Stoyan Nikolov

'Clinic of Surgical Diseases, University Hospital, Stara Zagora, Bulgaria

\begin{abstract}
Background: Colorectal cancer (CRC) is one of the most common malignancies in developed countries. Research for diagnostic and prognostic biomarkers is increasing. There are more than 750,000 papers indexed in PubMed directly related to biomarkers. A lot of these papers claim to report clinically useful biomarkers. Unfortunately, very few serum biomarkers are in clinical use. Materials and methods: We performed a review for most of the published CRC serum biomarkers in the PubMed, Embase and Web of Science databases. Results: In total, 119 articles were obtained from the databases, of which 89 articles reporting on CRC serum prognostic markers were considered as relevant. In these studies, a total of 200 individual tumor markers were obtained. Conclusions: A general approach to understand how to predict clinical outcomes using risk, diagnostic, and prognostic biomarkers, is needed. In the future, serum biomarkers will drive advances in risk, diagnosis, and prognosis, they will be the targets of powerful molecular therapies, and they will individualize and optimize therapy.
\end{abstract}

Keywords: cancer; biomarker; diagnosis; prediction; clinical outcome

\section{Introduction}

Colorectal cancer (CRC) is a worldwide oncological problem of high medical importance. Delineation of treatment approaches remains a challenge for the medical community. The wide spread of this disease continues to lead to an effort to improve prevention, prognostic assessment, subsequent treatment, quality of life and outcome in these patients. The diagnosis in many patients occurs too late in the advanced third and fourth stages of development. In these advanced stages complications and mortality are significantly higher, despite all the achievements of modern medical science and practice. It is anticipated that earlier detection or secondary prevention will permit more effective treatment with a better prognosis.

The growing number of scientific publications and the significant volume of new knowledge about the molecular and cellular mechanisms involved in the development, progression and metastasis of the CRC, as well as mechanisms of metabolism and excretion of xenobiotics, including applicable therapeutic agents provide a solid foundation and evidence for the introduction of new biological markers for screening, diagnosis, prognosis and prediction of treatment response in these widespread neoplasms.

A biomarker (or biological marker) is "any molecule or particle (incl. cell, tissue) that reflects the development of the disease process" [1].

The term "prognostic factor" is defined as any parameter which is estimated at diagnosis (or surgical treatment) and which is associated with the outcome of the disease (recurrence-free period or overall survival). The term "predictor" is a defined parameter, which may evaluate the therapeutic response or lack of response to a specific therapy.

Biomarker detection in serum or plasma has some drawbacks. One is the fact that in these fluids are a heterogeneous mixture of proteins derived from different tissues is found, making it difficult to attribute a differentially expressed protein to a tissue-specific disease. This limitation can result in the identification of putative protein markers that are not specific for CRC [2]. Furthermore, in serum a small number of major proteins (e.g., albumin) are highly concentrated and mask other less abundant proteins that could be interesting as biomarkers. One strategy to overcome this problem is to remove from the serum the most abundant proteins by using commercial affinity columns designed specifically for this purpose [3].

\footnotetext{
*Corresponding author: Emil Enchev, Clinic of Surgical Diseases, UMHAT "Prof. Dr. St. Kirkovich"AD, Stara Zagora, Bulgaria. Tel: +359894845263; +359884099549; Email: emo_19_89@abv.bg

Received 27 October 2017 Revised 19 December 2017 Accepted 26 December 2017 Published 30 December 2017

Citation: Yovchev Y, Enchev E, Dimitrov E, Minkov G, Nikolov S. Prognostic serum biomarkers for colorectal cancer - Review. J Cancer Res Ther. 2018; 6(1):1-5. DOI: 10.14312/2052-4994.2018-1

Copyright: @ 2018 Yovchev Y, et al. Published by NobleResearch Publishers This is an open-access article distributed under the terms of the Creative Commons Attribution License, which permits unrestricted use, distribution and reproduction in any medium, provided the original author and source are credited.
} 
In recent years, research on a global scale has attempted to define subsets of biochemical markers that may be useful predictors of response to treatment (evaluated through clinical response, toxicity, and time to disease progression) and prognostic markers to determine the aggressiveness of the disease and the likelihood of recurrence after surgery. The science of pharmacogenomics is emerging as an increasingly useful molecular tool to investigate the disparity in drug efficacy by analysis of patient variables such as genetic polymorphisms in drug targets, metabolizing enzymes, transporters, and influential receptors [4].

\section{Materials and methods}

A review for most of the published CRC prognostic serum biomarkers in the PubMed, Embase and Web of Science databases was performed.

\section{Results}

In total, 119 articles were obtained, of which 89 articles reporting on CRC serum prognostic markers were considered as relevant. In these studies, a total of 200 individual tumor markers were obtained.

\section{Carcinoembryonic antigen (CEA)}

CEA is one of the first biomarkers studied in CRC. CEA is a serum glycoprotein with a molecular weight of $180 \mathrm{kd}$ that is one of at least 19 related molecules that are members of the immunoglobulin gene superfamily. As such, CEA functions as a homotypic intercellular adhesion molecule that promotes the aggregation of human colorectal carcinoma cells [5]. CEA may facilitate metastasis of CRC cells to the liver and lung. CEA is a normal cell product that is overexpressed by adenocarcinomas, primarily of the colon, rectum, breast and lung [6]. Smokers have a higher circulating CEA concentration than non-smokers, but there are no significant effects of age, sex, or ethnic group on the normal range. The liver is the major site for clearance of CEA. Moderate to significant elevations of serum CEA can be observed in a variety of chronic and acute inflammatory diseases, including alcoholic cirrhosis, cholelithiasis, obstructive jaundice, cholangitis, liver abscess, emphysema, bronchitis, gastric ulcer, gastritis, diverticulitis, diabetes and collagen vascular diseases [7]. CEA level elevations are not unique to CRC, but are observed in several carcinomas. The majority of the preoperative CEA studies showed that it was an useful prognostic indicator. As a postoperative prognostic indicator following complete surgical resection of colon carcinoma, elevated plasma CEA levels should return to normal within four to six weeks [8]. Postoperative elevation of CEA level is an adverse prognostic indicator. Recently, CEA mRNA levels have also been found to be useful for the evaluation of CRC progression, with elevated postoperative CEA mRNA predicting the presence of micrometastasis [9]. Reducing the level of CEA after radical surgery is associated with increased possibilities for survival [10], and higher levels before and after neoadjuvant therapy may be useful in assessing associated with complete against the incomplete clinical response, despite the limited data [11].

A retrospective analysis of 109 patients who underwent neoadjuvant setting establish a decrease in the values of the CEA $<2.7 \mathrm{ng} / \mathrm{dL}$ after 4 weeks of radiotherapy, without to be statistically significant for the achievement of complete tumor regression $(95 \% \mathrm{Cl} 0.057-0.484, \mathrm{P}=0.001)$ [12] but levels of the marker $<0.5 \mathrm{ng} / \mathrm{dL}$ were significant predictor for clinical complete response and prolonged overall survival [13].

\section{CA 19-9}

The CA 19-9 assay measures a tumor-related mucin that contains the sialylated Lewis - a pentasaccharide epitope, lacto-N-fucopentose II [8]. CA 19-9 is produced by adenocarcinomas of the pancreas, stomach, gall bladder, colon, ovary and lung, and it is shed into the circulation. The upper limit of normal for healthy subjects has been defined by the cut-off value of $37.0 \mathrm{U} / \mathrm{mL}$ [14]. CA 19-9 has become an established marker for pancreatic cancer, but it is still regarded as a research test for CRC. Numerous studies have addressed the potential utility of CA 19-9 in adenocarcinoma of the colon and rectum. The reported incidence of elevated serum CA19-9 in CRC ranges from $20 \%$ to $40 \%$ [15]. The incidence of elevated CA $19-9$ is stage-related, with the highest sensitivity occurring in patients with metastases [16]. However, the sensitivity of CA 19-9 was always less than that of the CEA test for all stages of disease [17]. The false-positive rate $(>37.0 \mathrm{U} / \mathrm{mL})$ is $15 \%$ to $30 \%$ in patients with non-neoplastic diseases of the pancreas, liver and biliary tract. Consequently, CA 19-9 cannot be used for screening asymptomatic populations. Significant post-surgical decreases are observed for CA 19-9, but these decreases have not been correlated with survival or disease-free interval. However, the CA 19-9 abnormality preceded clinical manifestation of the disease in only $25 \%$ of the cases and provided a median lead time of only three months [18]. Serum CA 19-9 elevations maybe observed in as many as $20 \%$ to $40 \%$ of patients with latestage CRC, but cannot be regarded as a diagnostic aid nor can it be used to detect early stage disease. Progressive increases of the marker may signal disease progression in $25 \%$ of the patients who express the CA 19-9 marker, but this monitoring provides only a minimal lead time of one to three months. Monitoring with CA 19-9 has not been shown to improve the management of patients with CRC.

\section{CYFRA 21-1}

Cytokeratin 19 is a proven marker for identifying cancer cells as CYFRA 21-1 is a fragment of cytokeratin 19. When comparing the sensitivity and specificity of serum CYFRA 21-1 with that of the CAE and CA 19-9, it was found that the upper limit of CYFRA $21-1 \geq 1.13 \mathrm{ng} / \mathrm{ml}$, it has a sensitivity of $47 \%$, compared to $37 \%$ for CEA ( $\geq 3.05 \mathrm{ng} / \mathrm{m}$ ) and $32.6 \%$ for CA $19-9$ ( $\geq 23.1 \mathrm{ng} / \mathrm{ml})$ in early colorectal cancer. It was also observed correlation between Dukes' staging and the three serum tumor markers. In another study it was found that in the chemotherapy of colorectal cancer with 5-FU, the serum levels of CYFRA 21-1, and other molecular weight cytokeratins TPA (tissue polypeptide antigen) and TPS (tissue polypeptide specific antigen) and CEA and CA 19-9 are elevated in patients with poor treatment response and poor prognosis [19].

\section{Tissue inhibitor of metalloproteinase type 1 (TIMP-1)}

TIMP-1 is a multifunctional glycoprotein which inhibits most matrix metalloproteinases (MMPs). Several studies 
have compared the clinical significance of serum-plasma MMPs with TIMPs in the diagnosis of CRC, differentiation between CRC and colorectal adenomas, as well as their behavior in relation to clinico-pathological parameters of CRC and to classical tumor markers. The total levels of TIMP-1 in patients with CRC are significantly greater when compared to that of healthy blood donors who have a very narrow range of plasma TIMP-1 levels [20]. More importantly, TIMP-1 is capable of being detected at early stages of CRC. Conversely, plasma levels of total TIMP-1 in patients with colonic adenomas, inflammatory bowel disease or primary breast cancer, do not increase [21]. Preoperative TIMP-1 levels were proposed as a stage-independent prognostic biomarker for CRC in two independent studies [22]. The results of these studies, however, indicated that elevation of TIMP-1 was restricted to advanced stages of CRC. Additional studies are required to validate the use of TIMP-1 for both early diagnosis and evaluation of the prognosis of CRC.

The potential tumor marker impact of MMPs and TIMPs has been extensively studied. It was clearly shown by several authors that MMP-9 and TIMP-1 have significant potential as biomarkers in CRC. Diagnostic sensitivity of MMP-9 and TIMP-1 was consistently higher compared with those of conventional biomarkers (CEA or CA 19-9). Is has been suggested that MMP-9 and TIMP-1 estimation likely have the greatest predictive impact when screened as part of a biomarker panel [23].

In a very recent study, Wilson et al. [24] evaluated the accuracy of MMP-9 for CRC in an asymptomatic population. From 748 patients overall, 46 cases of CRC were identified. Univariate analysis showed that increased serum MMP-9 concentration, demographic characteristics and behavioral factors were all significantly associated with presence of CRC. The final logistic regression model had a sensitivity and specificity of $79 \%$ and $70 \%$, respectively.

Higher serum antigen concentrations of MMPs and TIMPs significantly correlated with tumor stage, nodal involvement and the presence of distant metastases. Previous results of tissue expressions concluded that MMPs and TIMPs play an important role in CRC invasion and metastasis, and they are also activated in premalignant colorectal adenomas. The increasing serum antigen concentrations of MMPs and TIMPs coincide with a multistep process of colonic carcinogenesis. Furthermore, it was suggested that measurement of MMPs and TIMPs might be useful in the assessment of preoperative tumor stage [25].

Several studies confirmed that high preoperative serum or plasma MMPs-2 and-9 and mainly TIMP-1 antigen levels are strong prognostic factors for patients with CRC and their determination might be useful for identification of patients with higher risk for cancer recurrence. Preoperative bloodlevels of TIMP-1 were independent predictors of diseasefree survival in patients with primary resectable CRC [26].

Very recently, Min et al. [27] showed that high serum levels of TIMP-1 were correlated with CRC liver metastasis and were significant predictive factors for poor prognosis following resection of synchronous liver metastasis.
In a pilot study, Pasternak et al. [28] measured MMPs in postoperative intraperitoneal fluid after rectal cancer surgery. They found that elevated MMPs- 8 and-9 levels were markers for later development of anastomotic leakage after surgery. They suggest that MMPs appear to have an important role in the development of anastomotic leakage and may be promising pharmacological targets to protect anastomotic integrity.

The immunoassay kits of MMPs and TIMPs are usually designed for determination of concentrations in cell culture supernates, serum and/or plasma. For quantitative comparison in humans, plasma and/or serum concentrations are acceptable to use, however the use of serum MMPs and TIMPs have been previously criticized due to its increased level compared with plasma estimation [29].

It is well known that MMPs are stored in macrophage and neutrophil granules, while most of TIMPs are secreted by platelets. Therefore, when using serum levels of MMPs and TIMPs, there are three to five fold higher levels than in corresponding EDTA or citrate plasma samples. Despite citrate plasma being the suggested sample of choice for estimating circulating MMPs or TIMPs [30], serum sampling may still be useful provided that methods of collection and processing were standardized [31]. Thus, one should be aware of the pre-analytical pitfalls to avoid misinterpretation of data when determining MMP and/ or TIMP levels. Further, when collecting samples it is recommended after centrifugation to an aliquot and store samples at $-20^{\circ} \mathrm{C}$ or $-80^{\circ} \mathrm{C}$ or assay immediately. The time elapsed between blood sampling and centrifugation is associated with higher serum MMPs levels, with a suggested seven-fold increase after $2 \mathrm{~h}$ [32]. MMPs degrades during storage, even at $-80^{\circ} \mathrm{C}$, therefore the repeated freeze-thaw cycles should be avoided, while TIMPs are stable and can be frozen/thawed for several times.

It has also been suggested that TIMPs can predict individual responses to chemotherapy. In the study of Sörensen et al. [33] ninety patients with metastatic CRC were included. Plasma TIMP-1 and serum CEA were measured in samples obtained before the first cycle of first-line combination chemotherapy. It was shown that plasma TIMP-1 concentrations obtained before the first cycle of chemotherapy were significantly and independently associated with objective response, time to progression (TTP) and overall survival (OS) of patients with metastatic $\mathrm{CRC}$ receiving combination of irinotecan, 5fluoruracil (5-FU), and folinic acid chemotherapy. CEA was not significantly associated with TTP or OS when TIMP-1 was included in the multivariable analysis. One explanation for the associations is that TIMP-1 protects cancer cells against the apoptotic stimuli that consecutively affect the cells [34].

\section{Serum MiRNA-21}

MicroRNAs (miRNAs) are endogenous, small non-coding RNAs with a length of 18-25 nucleotides. They were first reported in 1993 [35]. These miRNAs may regulate the translation of specific protein-coding genes [36]. Recent studies have shown revealed that overexpression of 
microRNA-21(miR-21) could increase cell proliferation, migration, invasion, and survival in a variety of cancer cell lines [37]. miR-21 was also found to be elevated in many cancers, including breast cancer, colon cancer, lung cancer, pancreatic cancer, prostate cancer, stomach cancer, hepatocellular carcinomas, ovarian cancer, and others [38]. Some studies have found overexpression of miR-21 to be closely associated with poor survival outcome in various cancers [39]. Higher levels of miR-21 expression have been found to be predictive of cancer outcome.

Elevated miR-21 expression was found to be predictive of poor survival among CRC patients in a meta-analysis [40]. The pooled HR of OS was 1.76 (95\% Cl: 1.34-2.32, $P=0.000$ ). The differences were found to be statistically significant, though the HRs were not strong. Hayes et al. reported that $H R>2$ was considered strongly predictive [41]. After elimination of heterogeneity, the pooled HR was 2.32(95\% Cl: $1.82-2.97, \mathrm{P}=0.000$ ), which was found to significantly predict poorer survival. Elevated levels of miR21 expression were found to have a prognostic role in CRC, but it was not possible to confirm miR-21 as independent predictive factor. Recently, researchers considered that a set of miRNAs might have a stronger predictive effect on survival than a single microRNA [42]. The expression of miR21 was detected in the samples of tumor tissue mostly, but few in serum or plasma in the selected studies. Circulating prognostic markers were found to be more valuable than tissue throughout the lives of the cancer patients.

Results from a study of an independent cohort of 186 CRC patients, 60 postoperative patients, 43 advanced adenoma patients, and 53 control subjects were evaluated [43]. In the validation cohort, miR-21 levels were statistically significantly elevated in preoperative serum from patients with adenomas $(P<0.001)$ and $C R C s(P<0.001)$. Importantly, miR-21 expression dropped in postoperative serum from patients who underwent curative surgery $(P<0.001)$. Serum miR-21 levels robustly distinguished adenoma (area under the curve $[\mathrm{AUC}]=0.813 ; 95 \%$ confidence interval $[\mathrm{Cl}]=0.691$ to 0.910 ) and CRC (AUC $=0.919 ; 95 \% \mathrm{Cl}=0.867$ to 0.958 ) patients from control subjects. High miR-21 expression in serum and tissue was statistically significantly associated with tumor size, distant metastasis, and poor survival.

Prognostic research has, to date, received much less attention than research into therapeutic or diagnostic areas, and an evidence-based approach to the design, conduct and reporting of primary studies of prognostic markers is needed [44]. Reviews have demonstrated that primary prognostic studies are often of poor quality [45]. Furthermore, synthesis of prognostic studies is a relatively new and evolving area in which the methods are less well developed than for reviews of therapeutic interventions or of diagnostic accuracy and available reviews have often been of poor quality [46]. For prognostic markers, apart from the duration of follow-up, the various aspects leading to observations of heterogeneity are almost similar to those for diagnostic markers.

\section{Conclusion}

Recently, there has been an impressive increase in the number of prognostic serum biomarkers in CRC, but yet it is not clear whether they have prognostic value or therapeutic implications. They may well be useful in taking decisions regarding the prognosis of CRC patients, but further prospective trials are clearly required. Identifying and understanding serum biomarkers can improve the effectiveness of treatment in several ways; for example, it can lead to the development of marker-specific therapies. Prognostic markers may also improve the selection of adjuvant therapies by identifying those who will benefit most and therefore avoid toxic side effects of treatment in patients with the least risk for recurrence. Furthermore, clinical predictions based on serum biomarkers will be an integral part of physician-patient-shared decision-making, and they will improve clinical care and patient outcomes. Novel noninvasive blood-based tests are strongly desirable for prognosis of the outcome in CRC.

\section{Conflicts of interest}

Authors declare no conflicts of interest.

\section{References}

[1] Agusti J, Herold S, Schwarz M, Sanchez P, Ljung K, et al. Strigolactone signaling is required for auxin-dependent stimulation of secondary growth in plants. Proc Natl Acad Sci U S A. 2011; 108(50):2024220247.

[2] Alvarez-Chaver P, Otero-Estévez O, Páez de la Cadena M, RodríguezBerrocal FJ, Martínez-Zorzano vs. Proteomics for discovery of CRC biomarkers. World J Gastroenterol. 2014; 20(14):3804-3824.

[3] Nibbe RK, Chance MR. Approaches to biomarkers in human colorectal cancer: looking back, to go forward. Biomark Med. 2009; 3(4):385396.

[4] McLeod HL, Yu J. Cancer pharmacogenomics: SNPs, chips, and the individual patient. Cancer Invest. 2003; 21(4):630-640.

[5] Benchimol S, Fuks A, Jothy S, Beauchemin N, Shirota K, et al. Carcinoembryonic antigen, a human tumor marker, functions as an intercellular adhesion molecule. Cell. 1989; 57(2):327-334.

[6] Ilantzis C, DeMarte L, Screaton RA, Stanners CP. Deregulated expression of the human tumor marker CEA and CEA family member CEACAM6 disrupts tissue architecture and blocks colonocyte differentiation. Neoplasia. 2002; 4(2):151-163.

[7] Sanders DS, Kerr MA. Lewis blood group and CEA related antigens; coexpressed cell-cell adhesion molecules with roles in the biological progression and dissemination of tumors. Mol Pathol. 1999; 52(4):174178.

[8] Fletcher RH. Carcinoembryonic antigen. Ann Intern Med. 1986; 104(1):66-73.

[9] Miura M, Ichikawa Y, Tanaka K, Kamiyama M, Hamaguchi Y, et al. Realtime PCR (TaqMan PCR) quantification of carcinoembryonic antigen (CEA) mRNA in the peripheral blood of colorectal cancer patients. Anticancer Res. 2003; 23(2B):1271-1276.

[10] Park YA, Lee KY, Kim NK, Baik SH, Sohn SK, et al. Prognostic effect of perioperative change of serum carcinoembryonic antigen level: A useful tool for detection of systemic recurrence in rectal cancer. Ann Surg Oncol. 2006; 13(5):645-650.

[11] Thirunavukarasu P, Sukumar S, Sathaiah M, Mahan M, Pragatheeshwar $K D$, et al. C-stage in colon cancer: implications of carcinoembryonic antigen biomarker in staging, prognosis, and management. J Natl Cancer Inst. 2011; 103(8):689-697.

[12] Jang NY, Kang SB, Kim DW, Kim JH, Lee KW, et al. The role of carcinoembryonic antigen after neoadjuvant chemoradiotherapy in patients with rectal cancer. Dis Colon Rectum. 2011; 54(2):245-252.

[13] Habr-Gama A, Gama-Rodrigues J, Perez RO. Is tailoring treatment of rectal cancer the only true benefit of long-course neoadjuvant chemoradiation Dis Colon Rectum. 2013; 56(2):264-266.

[14] Ritts RE Jr, Del Villano BC, Go VL, Herberman RB, Klug TL, et al. Initial clinical evaluation of an immunoradiometric assay for CA 19-9 using the $\mathrm{NCl}$ serum bank. Int J Cancer. 1984; 33(3):339-345.

[15] Gupta MK, Arciaga R, Bocci L, Tubbs R, Bukowski R, et al. Measurement of a monoclonal-antibody-defined antigen (CA19-9) in the sera of patients with malignant and nonmalignant diseases. Comparison with carcinoembryonic antigen. Cancer. 1985; 56(2):277-283. 
[16] lemura K, Moriya Y. A comparative analysis of the serum levels of NCCST-439, CEA and CA19- 9 in patients with colorectal carcinoma. Eur J Surg Oncol. 1993; 19(5):439-442. (PubMed)

[17] Thomas WM, Robertson JF, Price MR, Hardcastle JD. Failure of CA19-9 to detect asymptomatic colorectal carcinoma. Br J Cancer. 1991; 63(6):975-976.

[18] Ion D, Jinga D, Stoian RV, Bolocan A, Paduraru DN, et al. Arguments in favour of the compulsoriness of the imunohistochimic (IHC) exam in the colo-rectal cancer. IASGO Bucuresti. 2012.

[19] Holdenrieder S, Stieber P, Liska V, Treska V, Topolcan O, et al. Cytokeratin serum biomarkers in patients with colorectal cancer. Anticancer Res. 2012; 32(5):1971-1976.

[20] Holten-Andersen MN, Murphy G, Nielsen HJ, Pedersen AN, Christensen IJ, et al. Quantitation of TIMP-1 in plasma of healthy blood donors and patients with advanced cancer. Br J Cancer. 1999; 80(3-4):495-503.

[21] Holten-Andersen MN, Fenger C, Nielsen HJ, Rasmussen AS, Christensen IJ, et al. Plasma TIMP-1 in patients with colorectal adenomas: A prospective study. Eur J Cancer. 2004; 40(14):2159-2164.

[22] Holten-Andersen M, Christensen IJ, Nilbert M, Bendahl PO, Nielsen $\mathrm{H}$, et al. Association between preoperative plasma levels of tissue inhibitor of metalloproteinase 1 and rectal cancer patient survival. A validation study. Eur J Cancer. 2004; 40(1):64-72.

[23] Dragutinović WV, Radonjić NV, Petronijević ND, Tatić SB, Dimitrijević $\mathrm{IB}$, et al. Matrix metalloproteinase-2 (MMP-2) and -9 (MMP-9) in preoperative serum as independent prognostic markers in patients with colorectal cancer. Mol. Cell Biochem. 2011, 355, 173-178.

[24] Wilson S, Damery S, Stocken DD, Dowswell G, Holder R, et al. Serum matrix metalloproteinase 9 and colorectal neoplasia: A communitybased evaluation of a potential diagnostic test. Br J Cancer. 2012; 106(8):1431-1438.

[25] Hritz I, Varga MZ, Juhász M, Miheller P, Tulassay $Z$, et al. Increased serum MMP-2, MMP-7, MMP-9, TIMP-1 and TIMP-2 levels in colorectal cancer development. Gastroenterology. 2011; 140(5):S-343.

[26] Birgisson H, Nielsen HJ, Christensen IJ, Glimelius B, Brünner N. Preoperative TIMP-1 is an independent prognostic indicator in patients with primary colorectal cancer: A prospective validation study. Eur J Cancer. 2010; 46(18):3323-3331.

[27] Min BS, Kim NK, Jeong HC, Chung HC. High levels of serum VEGF and TIMP-1 are correlated with colon cancer liver metastasis and intrahepatic recurrence after liver resection. Oncol Lett. 2012; 4(1):123-130.

[28] Pasternak B, Matthiessen P, Jansson K, Andersson M, Aspenberg P. Elevated intraperitoneal matrix meetalloproteinases-8 and -9 in patients who develop anastomotic leakage after rectal cancer surgery: A pilot study. Colorectal Dis. 2010; 12(7):e93-e98.

[29] Jung K, Klotzek S, Stephan C, Mannello F, Lein M. Impact of blood sampling on the circulating matrix metalloproteinase $1,2,3,7,8$, and 9. Clin Chem. 2008; 54(4):772-773.

[30] Makowski GS, Rambsy ML. Use of citrate to minimize neutrophil matrix metalloproteinase-9 in human plasma. Anal Biochem. 2003; 322(2):283-286.

[31] Gerlach RF, Meschiari CA, Marcaccini AM, Palei AC, Sandrim VC, et al. Positive correlations between serum and plasma matrix metalloproteinase (MMP)-2 and MMP-9 levels in disease conditions. Clin Chem Lab Med. 2009; 47(7):888-891.

[32] Gerlach RF, Demacq C, Jung K, Tanus-Santos JE. Rapid separation of serum does not avoid artificially higher matrix metalloproteinase (MMP)-9 levels in serum versus plasma. Clin Biochem. 2007; 40(12):119-123.

[33] Sørensen NM, Byström P, Christensen IJ, Berglund A, Nielsen HJ, et al. TIMP-1 is significantly associated with objective response and survival in metastatic colorectal cancer patients receiving combination of irinotecan, 5-fluorouracil, and folinic acid. Clin Cancer Res. 2007; 13(14):4117-4122.

[34] Schneider J, Bitterlich N, Schulze G. Improved sensitivity in the diagnosis of gastro-intestinal tumors by fuzzy logic-based tumor marker profiles including the tumor M2-PK. Anticancer Res. 2005; 25(3A):1507-1515.

[35] Lee RC, Feinbaum RL, Ambros V. The C. elegans heterochronic gene lin-4 encodes small RNAs with antisense complementarity to lin-14. Cell. 1993; 75(5):843-854.

[36] Yang W, Lee DY, Ben-David Y. The role of microRNAs in tumor genesis and angiogenesis. Int J Physiol Pathophysiol Pharmacol. 2011; 3:140155.

[37] Lu Z, Liu M, Stribinskis V, Klinge CM, Ramos KS, et al. MicroRNA-21 promotes cell transformation by targeting the programmed cell death 4 gene. Oncogene. 2008; 27(31):4373-4379.

[38] Volinia S, Calin GA, Liu CG, Ambs S, Cimmino A, et al. A microRNA expression signature of human solid tumors defines cancer gene targets. Proc Natl Acad Sci U S A. 2006; 103(7):2257-2261.

[39] Chan SH, Wu CW, Li AF, Chi CW, Lin WC. miR-21 microRNA expression in human gastric carcinomas and its clinical association. Anticancer Res. 2008; 28(2A):907-911.

[40] Xia X, Yang B, Zhai X, Liu X, Shen K, et al. Prognostic Role of microRNA-21 in Colorectal Cancer: A Meta-Analysis. PLoS ONE. 2013; 8(11):e80426.

[41] Hayes DF, Isaacs C, Stearns V. Prognostic factors in breast cancer: current and new predictors of metastasis. J Mammary Gland Biol Neoplasia. 2001; 6(4):375-392.

[42] Li X, Zhang Y, Zhang Y, Ding J, Wu K, et al. Survival prediction of gastric cancer by a seven-microRNA signature. Gut. 2010; 59:579-585.

[43] Toiyama Y, Takahashi M, Hur K, Nagasaka T, Tanaka K, et al. Serum miR-21 as a diagnostic and prognostic biomarker in colorectal cancer. J Natl Cancer Inst. 2013; 105(12): 849-59.

[44] Altman DG, Riley RD. Primer: An evidence-based approach to prognostic markers. Nat Clin Pract Oncol. 2005; 2(9):466-472.

[45] Keough-Ryan T, Hutchinson T, MacGibbon B, Senecal M. Studies of prognostic factors in end-stage renal disease: An epidemiological and statistical critique. Am J Kidney Dis. 2002; 39(6):1196-1205.

[46] Kyzas PA, Loizou KT, loannidis JP. Selective reporting biases in cancer prognostic factor studies. J Natl Cancer Inst. 2005; 97(14):1043-1055. 